\title{
ARGOS: the laser guide star system for the LBT
}

\section{S. Rabien, N. Ageorges, L. Barl, U. Beckmann, T. Blümchen, et al.}

S. Rabien, N. Ageorges, L. Barl, U. Beckmann, T. Blümchen, M. Bonaglia, J. L. Borelli, J. Brynnel, L. Busoni, L. Carbonaro, R. Davies, M. Deysenroth, O. Durney, M. Elberich, S. Esposito, V. Gasho, W. Gässler, H. Gemperlein, R. Genzel, R. Green, M. Haug, M. L. Hart, P. Hubbard, S. Kanneganti, E. Masciadri, J. Noenickx, G. Orban de Xivry, D. Peter, A. Quirrenbach, M. Rademacher, H. W. Rix, P. Salinari, C. Schwab, J. Storm, L. Strüder, M. Thiel, G. Weigelt, J. Ziegleder, "ARGOS: the laser guide star system for the LBT," Proc. SPIE 7736, Adaptive Optics Systems II, 77360E (27 July 2010); doi: 10.1117/12.857210

SPIE Event: SPIE Astronomical Telescopes + Instrumentation, 2010, San Diego, California, United States 


\title{
ARGOS - The Laser Guide Star System for the LBT
}

\author{
S. Rabien ${ }^{\mathrm{a}}$, N. Ageorges ${ }^{\mathrm{a}}$, L. Barl ${ }^{\mathrm{a}}$, U. Beckmann ${ }^{\mathrm{j}}$, T. Blümchen ${ }^{\mathrm{e}}$, M. Bonaglia ${ }^{\mathrm{d}}$, J. L. Borelli ${ }^{\mathrm{e}}$, J. \\ Brynnel $^{\mathrm{c}}$, L. Busoni ${ }^{\mathrm{d}}$, L. Carbonaro ${ }^{\mathrm{d}}$, R. Davies ${ }^{\mathrm{a}}$, M. Deysenroth ${ }^{\mathrm{a}}$, O. Durney ${ }^{\mathrm{b}}$, M. Elberich ${ }^{\mathrm{j}}, \mathrm{S}$. \\ Esposito $^{d}$, V. Gasho ${ }^{b}$, W. Gässler ${ }^{\mathrm{e}}$, H. Gemperlein ${ }^{\mathrm{a}}$, R. Genzel ${ }^{\mathrm{a}}$, R. Green ${ }^{\mathrm{c}}$, M. Haug ${ }^{\mathrm{a}}$, M. Lloyd \\ Hart $^{\mathrm{b}}$, P. Hubbard ${ }^{\mathrm{b}}$, S. Kanneganti ${ }^{\mathrm{a}}$, E. Masciadri ${ }^{\mathrm{d}}$, J. Noenickx ${ }^{\mathrm{b}}$, G. Orban de Xivry ${ }^{\mathrm{a}}$, D. Peter ${ }^{\mathrm{e}}$, A. \\ Quirrenbach $^{\mathrm{g}}$, M. Rademacher ${ }^{\mathrm{b}}$, H. W. Rix ${ }^{\mathrm{e}}$, P. Salinari ${ }^{\mathrm{d}}$, C. Schwab ${ }^{\mathrm{g}}$, J. Storm ${ }^{\mathrm{h}}$, L. Strüder ${ }^{\mathrm{i}}$, M.

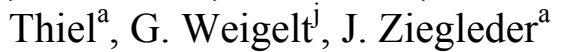 \\ ${ }^{a}$ Max Planck Institut für extraterrestrische Physik, 85748 Garching, Germany \\ ${ }^{\mathrm{b}}$ University of Arizona, Steward Observatory, Tucson, Arizona, USA \\ ${ }^{c}$ Large Binocular Telescope Observatory, Tucson, Arizona, USA \\ ${ }^{\mathrm{d} O s s e r v a t o r i o ~ A s t r o f i s i c o ~ d i ~ A r c e t r i, ~ F i r e n z e, ~ I t a l y ~}$ \\ ${ }^{\mathrm{e}}$ Max Planck Institut für Astronomie, Heidelberg, Germany \\ ${ }^{\mathrm{g}}$ Landessternwarte Heidelberg, Germany \\ ${ }^{\mathrm{h}}$ Astrophysikalisches Institut Potsdam, Germany \\ ${ }^{\mathrm{i}}$ Max Planck Institut Semiconductor Laboratory, Munich, Germany \\ ${ }^{\mathrm{j}}$ Max Planck Institut für Radioastronomie, Bonn, Germany
}

\begin{abstract}
ARGOS is the Laser Guide Star adaptive optics system for the Large Binocular Telescope. Aiming for a wide field adaptive optics correction, ARGOS will equip both sides of LBT with a multi laser beacon system and corresponding wavefront sensors, driving LBT's adaptive secondary mirrors. Utilizing high power pulsed green lasers the artificial beacons are generated via Rayleigh scattering in earth's atmosphere. ARGOS will project a set of three guide stars above each of LBT's mirrors in a wide constellation. The returning scattered light, sensitive particular to the turbulence close to ground, is detected in a gated wavefront sensor system. Measuring and correcting the ground layers of the optical distortions enables ARGOS to achieve a correction over a very wide field of view. Taking advantage of this wide field correction, the science that can be done with the multi object spectrographs LUCIFER will be boosted by higher spatial resolution and strongly enhanced flux for spectroscopy. Apart from the wide field correction ARGOS delivers in its ground layer mode, we foresee a diffraction limited operation with a hybrid Sodium laser Rayleigh beacon combination.
\end{abstract}

Keywords: Laser Guide Stars, Ground Layer Adaptive Optics, Large Binocular Telescope

\section{INTRODUCTION}

The basic motivation for the implementation of ARGOS at the LBT telescope is driven by the science goals that can be achieved with the system. The GLAO correction that ARGOS will provide for both LUCIFER [1] instruments, consisting of wide field multi object spectrographs [2] and NIR imagers, will result in a major gain for lots of scientific programmes. The ability of the laser guided ground layer adaptive optics to shrink the PSF size in almost all seeing conditions and over a wide field of view makes it possible to conduct observations in much shorter time, and even enables several cases that cannot be done without laser guided adaptive optics. Derived from the science cases the major goals of ARGOS can be condensed in two lines:

- Enhance the image quality and ensquared energy by a factor 2-3 for the LUCIFER instruments

- Implement a reliable, low maintenance and robust system with high observing efficiency

The technology that we will implement at LBT for ARGOS utilizes powerful green pulsed lasers to form a constellation of beacons on sky. The light from the scattered photons reaching back to the telescope will be split off by dichroic beam

Adaptive Optics Systems II, edited by Brent L. Ellerbroek, Michael Hart, Norbert Hubin, Peter L. Wizinowich, Proc. of SPIE Vol. 7736, 77360E - C 2010 SPIE · CCC code: 0277-786X/10/\$18 - doi: 10.1117/12.857210 
splitters in front of LUCIFER and sent to the ARGOS wavefront sensors. The Hartmann sensor itself uses an optical shutter to gate out the desired slice of the atmosphere and to detect the photons on a fast, large frame and low noise detector.

Great progress and first science programmes are currently being achieved with LUCIFER I on the mountain. In Fig. 1 Kband spectra of several distant star forming galaxies are shown, giving a preliminary view of the capabilities of the LUCIFER MOS unit on sky. With ARGOS 2-3 times spatially sharper observations will be possible, resulting as well in a 4-9 times shorter integration times, or accordingly deeper images and spectra.

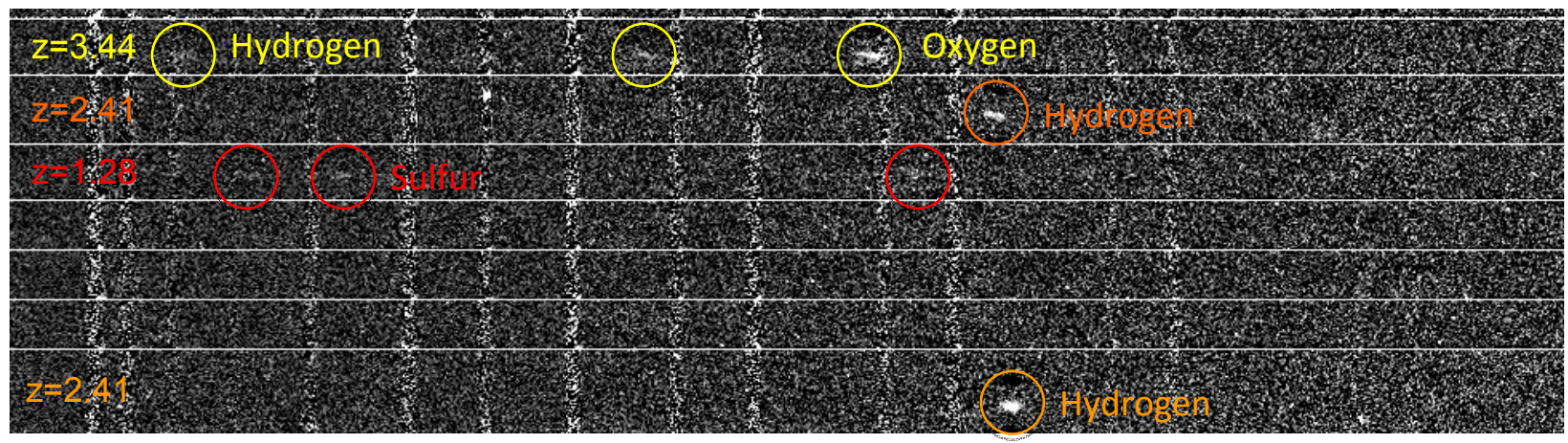

Fig. 1: The press release image from the LUCIFER spectrograph: NIR K-band spectra of distant star forming galaxies. Several emission lines of various chemical elements are detected. These emission lines are extremely faint and can only be seen after several hours of integration time even with the LBT's eight meter mirrors. The multiplexing capability of the LUCIFER MOS instrument allows for efficient observing of up to twenty galaxies in one pointing. Here only a few of the observed spectra in one frame are shown.

\section{SCIENCE DRIVERS OF GROUND LAYER ADAPTIVE OPTICS}

The prime driver for the ARGOS ground layer adaptive optics system (GLAO) is to greatly enhance the science that can be done on existing LBT facility instruments, and in particular with LUCIFER. It will enable this by improving the resolution and sensitivity for both imaging and multi-object spectroscopy over a very wide field of view. In particular, the combination of GLAO with a wide field MOS will be a unique facility.

The direct benefits to the LBT are afforded by the factor of 2-3 improvement in the spatial resolution. Indeed, ARGOS can be considered as a 'seeing enhancer' for the existing facility instruments that enables one to address much more of the primary science that has been identified in their respective science cases. This is because:

- observations can be done much faster, saving a significant amount of observing time.

- demanding science programmes, that would normally require the best seeing conditions, can instead be carried out during most nights.

Upgrade paths include enabling operation with other instruments, specifically LBTI, the thermal IR interferometer. Additionally, the upgrade path to diffraction limited resolution will provide a further benefit in terms of opening up new science with LUCIFER that could not previously have been addressed, and several science cases are connected with that capability.

The implementation plans for laser guide star adaptive optics followed by most 8 -m class observatories - specifically including Keck[5], VLT [6][7], Subaru [8], and Gemini [9]- have begun with a single sodium laser and single-conjugate adaptive optics. This provides diffraction limited performance over a rather small ( $<20$ ") field of view. Such LGS-AO observations have been proceeding for several years at those telescopes.

In contrast to this, ARGOS aims to provide enhanced resolution and sensitivity for both imaging and multi-object spectroscopy over a very wide field of view. ARGOS will provide a resolution comparable to that of HST/NICMOS (0.2arcsec in the K-band) over a full 4arcmin field of view. This remarkable performance will greatly boost the 
capabilities of LUCIFER, the instrument for which it is primarily conceived. Indeed, it is the increase in speed (to reach a given signal-to-noise) for LUCIFER's wide field MOS capability that makes GLAO such a compelling choice for the LBT. The complementarities of this combination to currently operational AO systems will make near infrared observations on the LBT extremely competitive with those attainable at other world-class observatories.

An indication of the performance that can be expected is demonstrated by Fig. 2 This image shows the central 700 pc of the nearby Seyfert galaxy NGC 4945 (D 4 Mpc) and was obtained with the VLT LGSF and NACO. It is at an angular resolution ( 0.15 ") comparable to that predicted for the LBT LGS-GLAO in good conditions, and proves sufficient to resolve individual stars. Note, however, that the field of view that LUCIFER will cover at such resolution is about 40 times greater. The brightest stars in Fig. 2 have a magnitude of $\mathrm{m}_{\mathrm{K}}=17.5$ to 18.0 which, for a distance modulus of 28.0, yields an absolute magnitude of $\mathrm{M}_{\mathrm{K}}=-10$ to -10.5. This implies that they are in fact late type supergiants, since KI stars have visual magnitudes of $\mathrm{M}_{\mathrm{V}}=-7.5$ and colours of $\mathrm{V}-\mathrm{K}=2.5$. Because such stars only occur for a short while about $10 \mathrm{Myr}$ after a burst of star formation, direct observations of so many of them indicate unambiguously that there has been recent star formation in the nuclear region of NGC4945. Photometry of the clusters within a few arcsec of the nucleus suggests that in each cluster there may be ten to a few tens of such stars, suggesting that the star formation here has been very intense and vigorous. With GLAO on the LBT it would be possible to study in equivalent detail a number of other very nearby galaxies - such as Circinus and Centaurus A - which play an important role in our understanding of the impact of stellar processes on galaxy evolution.

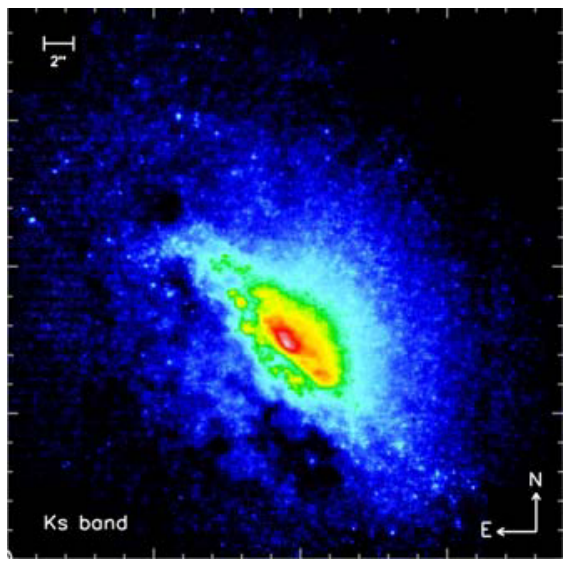

Fig. 2: K-band image of NGC4945 taken with LGS-AO at the VLT. This galaxy is at a distance of about 4Mpc and yet in this image it is possible to resolve individual stars. The data have a resolution of about 0.14 ", similar to that which will be achievable with LGS-GLAO on the LBT in good conditions; but the field is much smaller, only 37 "x37" in comparison to the 240"x240" available with LUCIFER.

Apart from the case indicated above a wide range of science will strongly benefit by the enhanced resolution and ensquared energy that ARGOS delivers. Amongst others the scientific topics that can be addressed with the aid of GLAO span a wide range from extragalactic cases such as high-z galaxy dynamics, AGN and QSO host galaxies, to galactic astrophysical questions about planets, cepheids or stellar clusters. With the GLAO system a major gain in resolution over a wide seeing range can be achieved, thus decreasing integration times required by large factors.

\section{GROUND LAYER ADAPTIVE OPTICS}

Proposed in 2001 [3], ground layer adaptive optics aims to correct the disturbances arising from the part of the earth's atmosphere close to the ground. While with this method the turbulence at high altitude stays uncorrected and thus the diffraction limit of the telescope is not reached, GLAO is associated with some benefits that normal single conjugated adaptive optics can not deliver: The wide field of view where a correction can be achieved and the uniform image quality over that field. Selecting and correcting the ground layer requires the higher layer contribution in the wavefront sensing to be excluded by some means. Either multiple natural guide stars can be selected over a large field, with the average of the wavefronts containing only little high layer contribution; or one can use laser guide stars and specifically choose to 
create them at low altitude. GLAO with natural guide stars has been demonstrated to work well with ESO's MAD instrument [10]. GLAO systems using a single Rayleigh guide star are in operation or development at the SOAR [13] and at the WHT [12]. Multiple beacon LGS GLAO has recently been demonstrated at the MMT [14].

For ARGOS we have chosen to implement a multi guide star system using low altitude Rayleigh stars. Because the system is independent of natural asterisms, we can achieve a uniform and reliable correction over a field as large as 4 by 4 arcmin. The actual performance of a ground layer adaptive optics system depends of course on the fraction of turbulence power being present within the first kilometres above the telescope. To calculate the performance of our system we utilize turbulence models that are based on measurements that have been carried out on mount Graham [11]. Those measurements do show that a significant fraction of the cumulative turbulence power is located close to the ground, well reachable by GLAO.

As we intend to run the GLAO under almost every atmospheric condition we evaluate the system performance with multiple turbulence profiles, representing good, medium and bad turbulence. Monte Carlo simulations have been carried out by generating numerous instances of atmospheric phase screens and imaging stars and lasers through those. Using novel tools that implement optics ray tracing into the adaptive optics closed loop models allows us even to track the performance including the optics models, aberrations or flexure.

While in the early calculations the faster and easier open loop simulation was used, we since then updated the simulation code to closed-loop control. It is interesting to note that there is no major difference in the outcome from both computation methods. The reason for this is in the relatively large residuals from high layer turbulence that mainly dominate the error budget.

The closed-loop simulations confirm our earlier results that the GLAO correction improves the FHWM by a factor 2-3. A summary of multiple performance calculations is shown in Fig. 3.

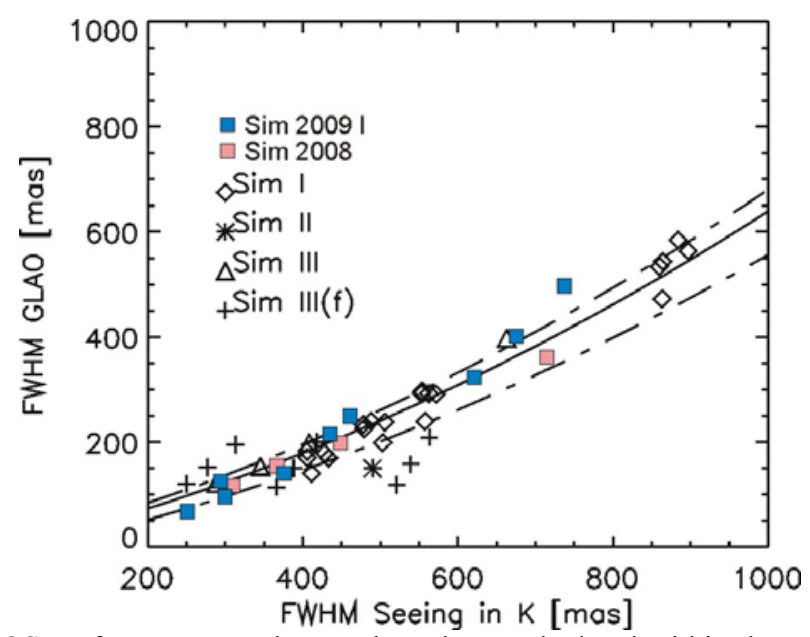

Fig. 3: summary of the ARGOS performance numbers as have been calculated within the various project phases. In black and white the points from the phase A study are given. In pink the results with inclusion of more effects from PDR are shown. With the updated closed loop simulation the blue points results. Basically we expect an improvement between 2-3 in FWHM and accordingly in the energy coupled to the MOS slits. 


\section{THE LBT LASER AO SYSTEM}

ARGOS is based on laser beacons created with Rayleigh scattering from air molecules, generated with multiple high power pulsed green lasers. Upon a trigger command all laser heads are firing synchronized a $~ 40$ ns wide light pulse. Within the laser systems the beams are first expanded to some mm width and then directed to a pupil mirror at the lower end of the launch telescope. With the optics in the laser box the pointing direction and the positioning of the laser beams is performed and as well the required polarization adjustment.

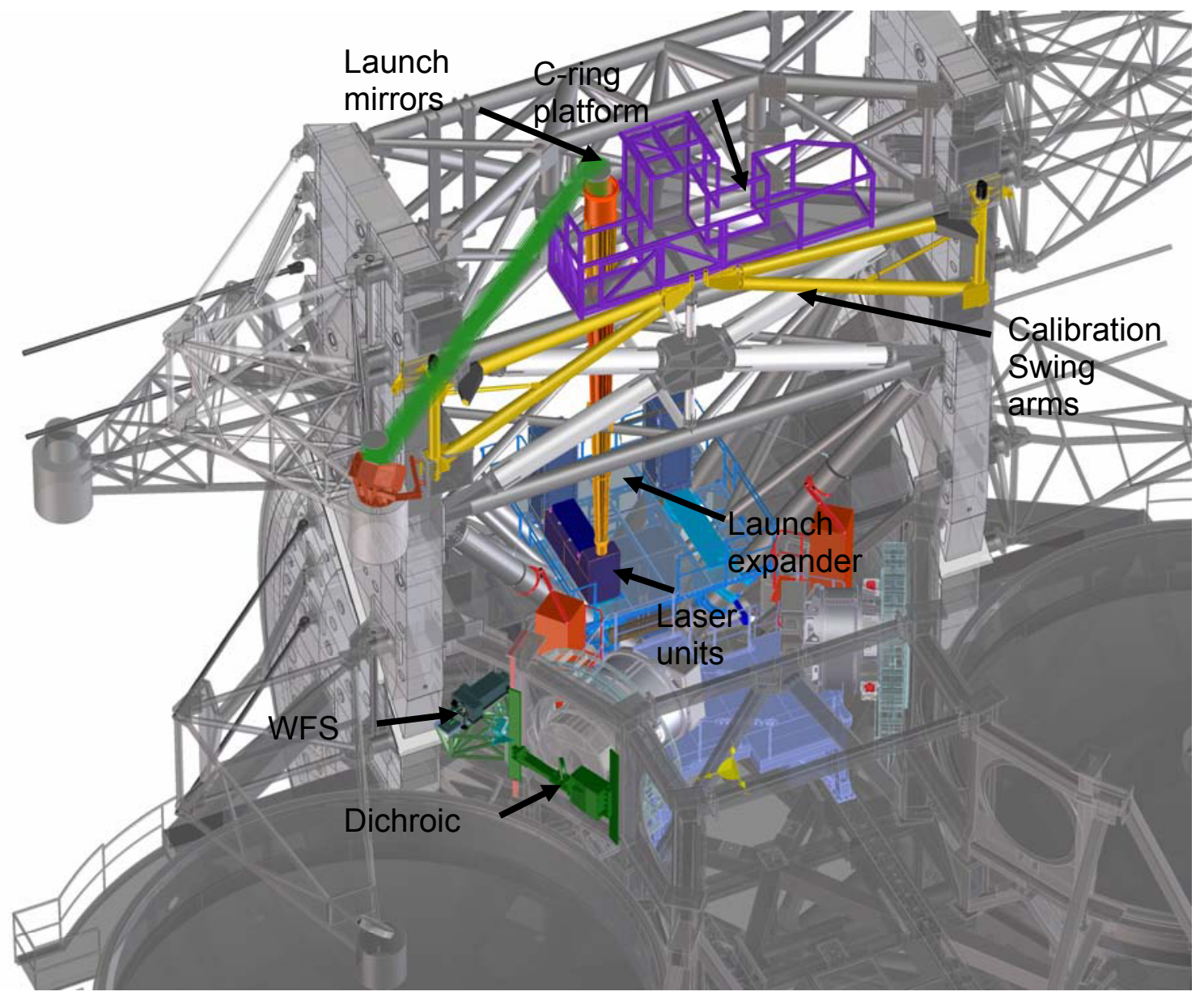

Fig. 4: overview of the ARGOS components as will be installed at the LBT. In the center piece between the two mirrors the laser units will be installed. Dedicated platforms allow access and servicing those units. From that location on the laser beams are expanded with a refractive beam expander being built into the LBT structure. With large flat mirrors the beams are directed to behind the secondary mirror and sent to sky. When coming back, the photons are split of with a large dichroic mirror, sending the light to the wavefront sensor. Additional swing arms allow to move calibration light sources and optics into the Gregorian prime focus, enabling us to calibrate the adaptive optics during daytime.

Travelling through the launch telescope, the beams are expanded and focused to a $12 \mathrm{~km}$ distance. After the refractive beam expander two large fold mirrors send the beams to behind the secondary and from there towards sky. While travelling through atmosphere, some photons out of the pulse will be scattered by air molecules. After $80.06 \mu$ s photons scattered at a $12 \mathrm{~km}$ distance arrive back again at the telescope.

In front of the instrument rotator structure we separate off the laser photons with a dichroic beam splitter and direct the light towards the ARGOS wavefront sensors. Inside the WFS the beams are first collimated and brought a bit closer to each other. A piezo driven mirror enables a fast correction of the uplink and vibration induced jitter. In the following the 
Pockels cells gating units slice out exactly those photons being scattered at $12 \mathrm{~km}$ distance within a $300 \mathrm{~m}$ range. The light out of this limited volume then falls through the lenslet array onto the detector. The detector itself is a fast large frame PnCCD allowing all three laser guide stars to be imaged on a single frame. With the lasers running at a $10 \mathrm{kHz}$ repetition rate, the Pockels cells will be triggered at the same rate. The detector accumulates the photon bunches from $\sim$ ten pulses and is read out then.

The frames from the CCD are transferred to the Argos slope computing units. This computer calculates the centroid position of all spots and sends the resulting slope vector to the adaptive secondary mirror where the reconstruction is performed in dedicated fast parallel computers, and the thin shell of the adaptive M2 mirror is set.

Since the laser guide star position as measured on sky does not reflect the atmospheric tilt properly, a separate tip-tilt sensor is required. With calculating the performance of several possibilities, an APD based system was chosen as best performing. The light for this APD quad cell is picked in the rotator structure on the so-called w-unit of the first light AO. Additionally some small amount of the natural guide star light is directed to the pyramid wavefront sensor that is already located on that board. This signal will be used to sense 'true' $\sim 10$ modes and slowly offset the laser guide star wavefront slopes. The purpose of this truth sensing is to adapt for non-common path aberrations, imperfect calibrations and the slight difference between elongated spots on sky and round calibration spots.

\section{LASER AND LAUNCH SYSTEM}

Within the ARGOS laser units the laser pulses are generated, directed to the proper constellation positions on sky, stabilized for flexure deviations, compensated for vibration induced jitter and adopted to the right polarization plane. Two of those laser systems will be mounted to the LBT structure. Within each of the systems three laser heads are arranged. The lasers themselves are produced by Innolas, consisting of frequency doubled Nd:YAG systems lasing at $532 \mathrm{~nm}$. With emitting more than $18 \mathrm{~W}$ at a pulse repetition rate of $10 \mathrm{kHz}$ and a pulsewidth less $40 \mathrm{~ns}$, the photon flux will be more then sufficient to generate bright guide stars. When the laser beams leave the heads a lens assembly pre-expands the beams to a $5 \mathrm{~mm}$ and ensures common collimation. As in the long train after the laser exit window several mirror surfaces and geometric rotations are present, we include a polarization adopting device based on an uniaxial crystal. With this device we ensure that the polarization planes of the guide stars on sky match the receiver acceptance direction. In the following a motorized periscope assembly allows to steer the beams according to an on sky field of 1 arcmin. While the beam position inside the laser box is actively locked to a position on a CCD, the offsets to the definition position are sent to the laser system from the wavefront sensor, ensuring perfect centring. At the location where the three beams join in the pupil plane of the launch telescope, we fore see a fast PZT driven mirror. With this mirror vibration induced beam jitter in the upwards path will be compensated. Additionally several diagnostics aid the operator for checking the laser power, the pulse duration and timing as well for delivering housekeeping information. A CAD view of the system is shown in Fig. 5. A detailed description of the laser system is presented in [17] within this proceedings.
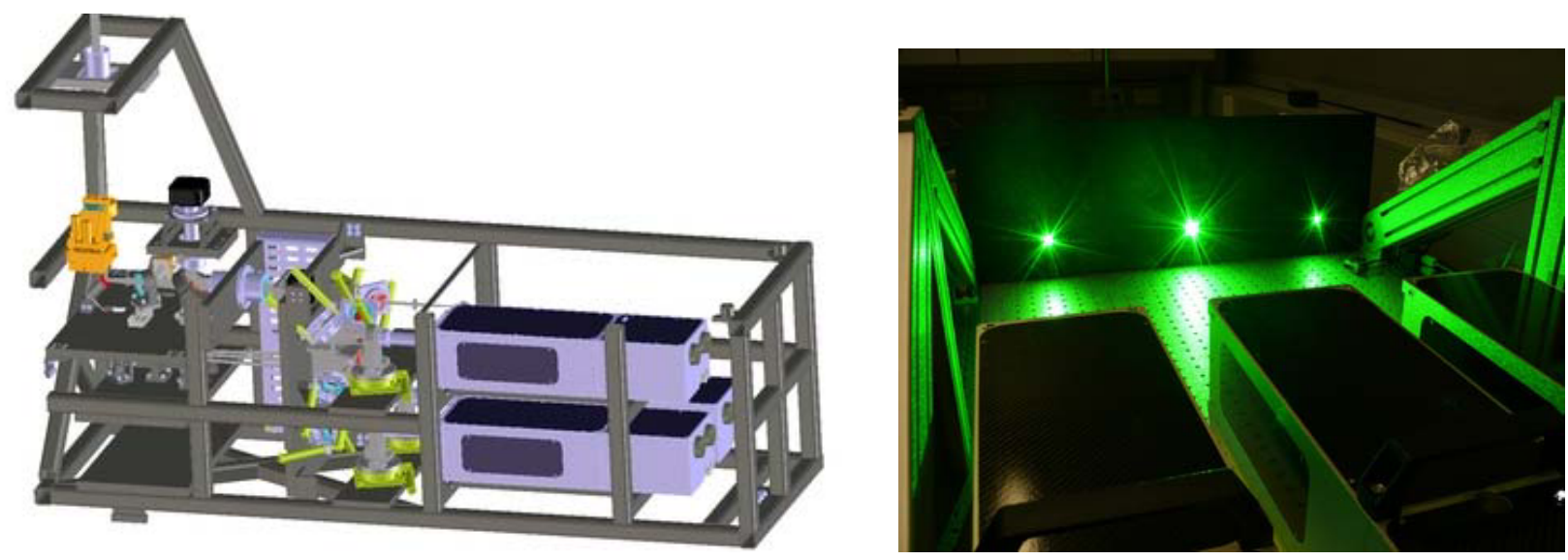

Fig. 5: CAD view of the Laser system frame with some of the contents shown. To the right in the system the laser heads are located. In the beam train the polarization controllers, the position steering and multiple diagnostic tools are located. On top the laser beams leave the system and are widened to the appropriate size in the launch expander. In the right picture three of the laser heads are shown when simultaneously firing at full power. The heads are currently under extensively test at MPE. 
Following the exit of the laser system, the launch system has the task to widen the beam at its exit and focus the beacons down to a small as possible spot in $12 \mathrm{~km}$ distance. Additionally larger range flexures can be compensated by the system with moving one of the large mirrors. For the expander a refractive design has been chosen with a $40 \mathrm{~cm}$ clear aperture at the large lens position. The focal length is quite large and expands over the distance between the laser instrument platform and the top end of LBT exhibiting quite loose tolerances for simplified alignment. The whole expander, from the laser system to the large lens, is fully sealed and enclosed. This protects against dust and bugs and ensures a long lived save operation. As an additional measure for dust protection we foresee shutters that are being closed over the larger optics when the laser is not in use. With the aim to produce the smallest spot on sky possible the optical demands on the large optics are quite high. An additional constraint is the allowed weight on top of the LBT M2 mirror, calling for a lightweight construction and lightweight mirrors. With using the hextex fusion honeycomb mirrors the whole unit on M2 top including the shutters and stiff mounting weights less then $120 \mathrm{Kg}$.
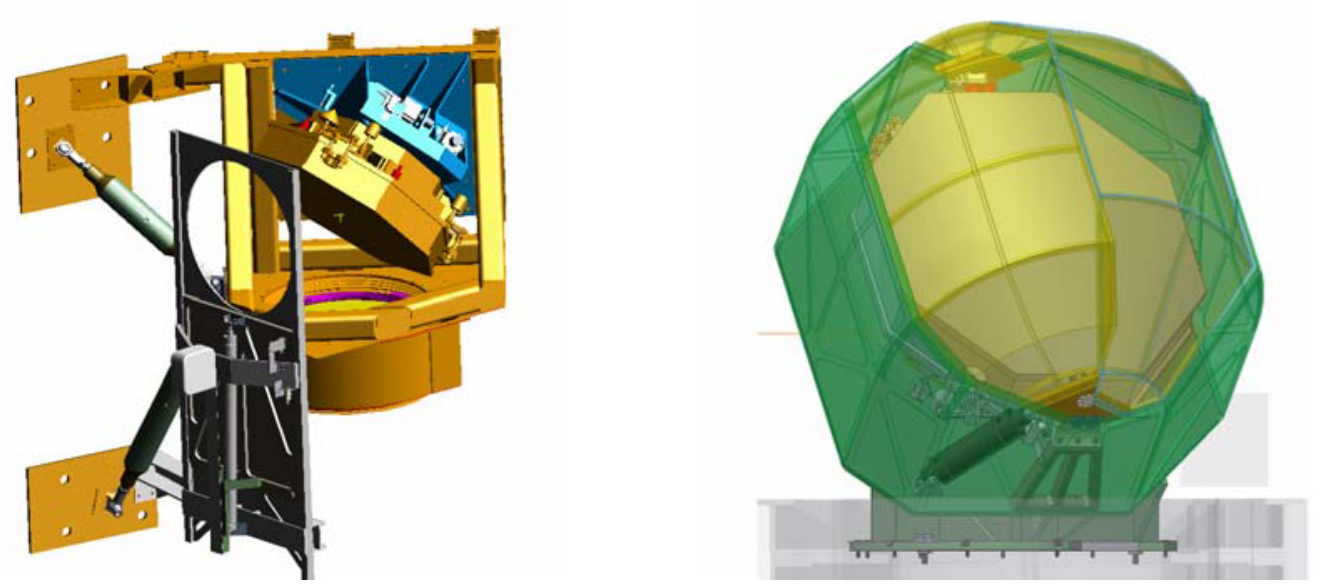

Fig. 6: parts of the laser launch system. To the left the top part of the expander with the $400 \mathrm{~mm}$ lens in its holder is shown. This piece will be mounted into the top steel structure of the LBT. The flat mirror seen on top of the lens directs the light to above M2 and can be steered remotely for alignment. On the right the $\sim 700 \mathrm{~mm}$ mirror design on top of M2 in its housing and dust cover is shown.

\section{WAVEFRONT SENSORS}

Detection of the backscattered light from the laser beacons and measurement of the wavefront is done in a Shack Hartmann sensor setup. We foresee one wavefront sensor for each of the LBT eyes collecting the light of three guidestars. This sensor will be mounted at a spare focal station of the LBT, aside of the scientific instrument.

Picking off the laser light takes place in front of the instrument rotator structure with a dichroic reflector, passing the light longwards of $\sim 600 \mathrm{~nm}$ to the NGS wavefront sensor, tip-tilt detector and the scientific instrument. (For a description of the LBT's NGS ,first light adaptive optics' please refer to [15] and [16] within this volume) This dichroic reflector has an optimized coating with minimum thermal impact for the scientific instrument. The shape of the dichroic is optimized with one cylindrical surface to correct the aberrations it would induce.

The optical setup of the wavefront sensors consists of a collimating lens after the Rayleigh focal plane, a Pockels cell unit for fast shuttering the light, re-imaging and collimating optics and the common lenslet array in front of the CCD.

The periscope mirrors in this design serve to get the light of all guide stars closer to the symmetry axis. Additionally the second mirror is located in a pupil plane and mounted on a fast piezo tip-tilt platform. With this mirror the Shack Hartmann spots will be stabilized within their sub-apertures so any laser beam jitter on the up-link can be compensated fast and reliably.

The Pockels cell assembly follows immediately after the pupil plane. This device will open with the repetition rate of the laser firing after the appropriate time of flight. Optically, we will custom assemble the cell out of a polarizer pair, and electro-optic crystals in a newly invented setup at MPE. The test performance of those devices shows a 1:5000 supression rate over a wide field of view. 


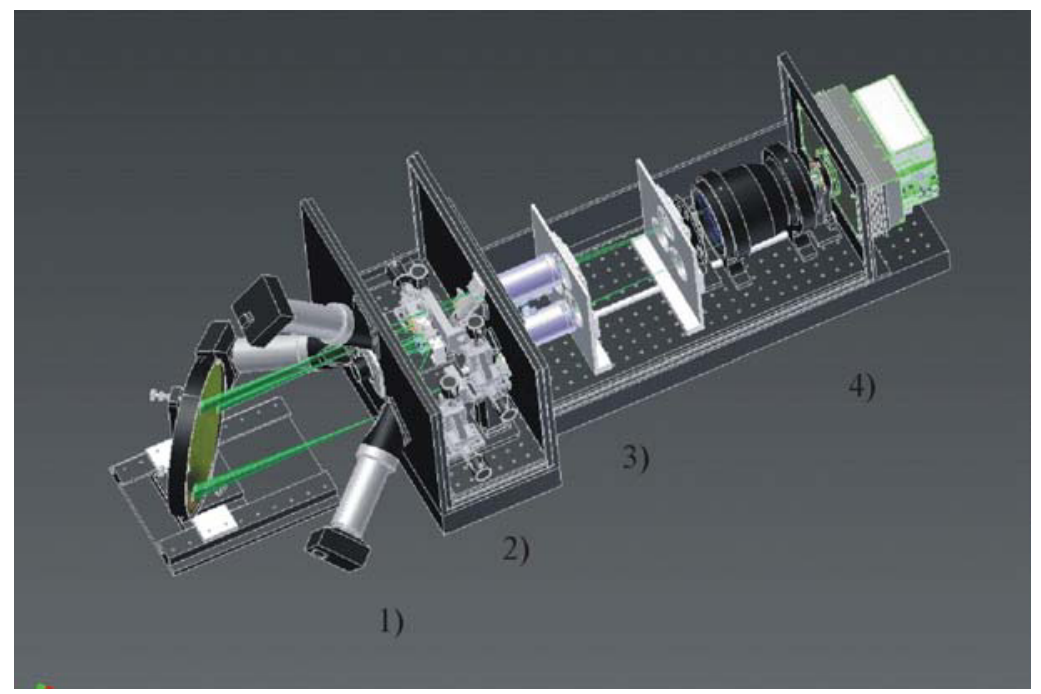

Fig. 7: view onto the mechanical arrangement of the WFS setup. A steel structure will mount it stiffly to the LBT gallery. Inside the dry air flushed cover the main elements can be seen: 1) patrol cameras 2) periscope \& TT, 3) Pockels cells 4) collimator lenslet \& detector

The re-focussing lens, the field lens and the common collimator together re-image then the pupil onto a lenslet array close in front of the detector.

The CCD itself is manufactured at the MPI semiconductor laboratories and offers a 264x264 pixels at a frame rate of $1 \mathrm{kHz}$, less then $3 \mathrm{e}^{-}$readnoise and a quantum efficiency close to one. A test detector is currently in operation at MPE. The next evaluation model is announced for April 2010 with the inclusion of a peltier cooler and a completely new compact housing. More details of the detector are presented in [18] in this proceedings.

To assist the acquisition and speed up this process we plan to implement cameras looking at the Rayleigh beacon focal plane. Those cameras allow the beacons to be located within a one arcmin field. So in the case of beacons being off the entrance field stop (e.g. after slewing to a new observation position) an automatic loop tracks them back to the capture range of the WFS. The detailed design of the wavefront sensor system is described in [19] within this proceedings

\section{TIP-TILT \& TRUTH CONTROL}

The position of a laser beacon on sky, as seen with the wavefront sensors, does not represent the atmospheric tilt properly. To measure the real image motion still a natural guide star is required. Aiming for as faint as possible stars to serve as a tilt reference, we will implement read noise free detection. Especially two possibilities have been compared: the detection with an APD quad-cell setup and the implementation of an electron multiplied CCD. While the latter would have offered the possibility to easily detect some low order modes in parallel and serve as a 'truth' sensor, the first possibility turned out to perform better in terms of limiting magnitude. The difference in performance is mainly caused by the so-called 'clock induced charge' and plays a role when using multiple pixels for the tilt determination. 


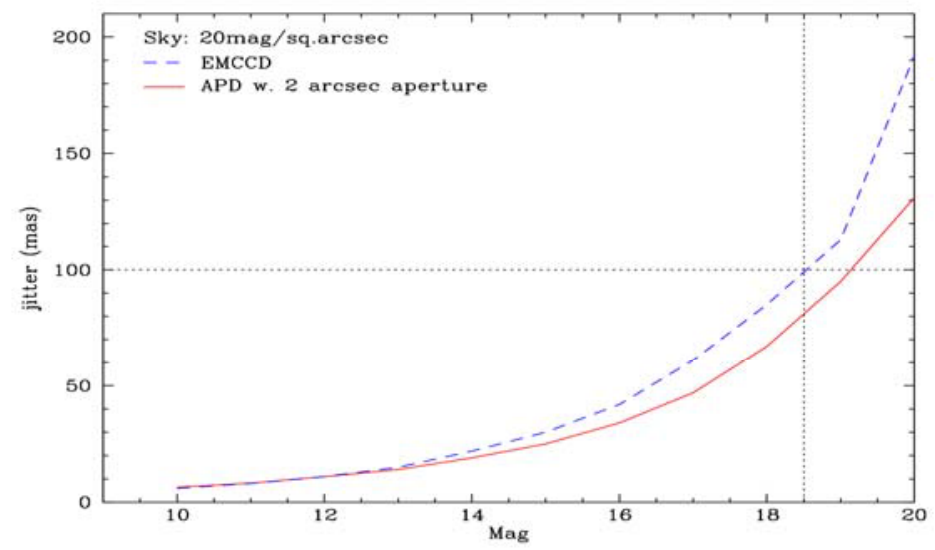

Fig. 8: performance comparison between a EMCCD based tip-tilt tracker and an APD quadcell solution. Shown is the RMS image motion in dependence of the tilt star magnitude. With the ground layer adaptive optics requiring a jitter less then 100 mas, we should be able to track on an 18.5 mag star.

Fig. 8 shows the performance of an EMCCD vs. the APD solution. With the APD performing slightly better, we decided to use this solution. The TT tracker is based on quadrant detection with a lens array feeding optical fibres. At the end of those fibres commercial APD units are placed, being located in an electronic rack, away from the telescope environment. Mechanically the feed head will sit on the w-board of the first light AO, utilizing space available in front of the acquisition CCD. Electronically we will implement stand-alone electronics that counts the photons in the quadrants, calculates the centroids and sends a vector with the star location to the ARGOS slope computing units.

As the quadrant sensor only sees the tip and tilt of the wavefront, some low order modes on a natural guide star must be sensed elsewhere. This task now is assigned to the pyramid sensor of the first light AO. It will measure slowly the first $\sim 10$ modes of the wavefront, such that any flexure or otherwise induced non-common path aberrations between the LGS sensor and the science instrument are eliminated.

\section{CONTROL SYSTEM}

As adaptive optics is mainly a 'control issue' the real time computing and control of the wavefront is a major ingredient for success. Fig. 9 shows an overview of the ARGOS control scheme. More information on the control strategy is given by [20] in this proceedings. In addition to the real time and instrument control, the ARGOS system will provide the user with information on the resulting PSF shape, assisting greatly the science data reduction. A discussion of this topic is given by [21] in this volume. Multiple loops in the system care for the laser and launch, the adaptive optics loop and the natural guide star tilt and truth control. In the following some of the loops are described:

\subsection{Laser and launch control:}

Laser System Field Control and Pupil Control: An arrangement of two tip tilt mirrors in a periscope setup, separate for each laser, provide the ability to adjust for differential pointing. The positions of the lasers in the focal plane are measured with one field diagnostic camera and the positions of the lasers in the pupil plane are measured with one pupil diagnostic camera. Changes in the positions, i.e. due to flexure are compensated in closed loop.

Laser Launch Stabilization Control: with vibrations being present and jittering the laser beacons on sky, we foresee compensation with open loop accelerometer measurements at the launch telescope. This control is built with a dedicated real-time computer and runs at $\mathrm{kHz}$ rate.

Laser Launch Focus Control: In the long term the laser launch focus should be controlled by a lookup table in feed forward with temperature as input and related positions as output. As this will not be available at the beginning and to have a way to control the laser focus on sky the LGS wavefront data is used for focus evaluation. The FWHM of the Shack-Hartmann spots is used to identify changes in focus and optimize the focus of the laser spots. 


\subsection{Laser Guide Star WFS control loops}

LGSW Field Steering Control: A patrol camera in the WFS monitors and positions the laser on the LGS WFS initially. This tip and tilt is controlled with a periscope mirror system within each sensor path. This tilt is applied in a high rate to a PZT mirror to keep the laser beams in position and parallel. Long term drift in tilt eating up the dynamic range is offloaded to the laser system field and pupil control.

LGSW Pupil Tracking Control: The pupil position on the WFS is tracked by calculating the intensity distribution of the SH pattern. The intensity of each sub-aperture is calculated by summing the counts of all pixels and the centroid of all sub-apertures is calculated. A lens after the Pockels cells is used to correct for any centre offset.

LGSW Focus Control: The focus offset measured with the Truth sensor is offloaded via the wavefront data handling to the WFS and registered as slope offset. Large long term de-focus terms can be reduced by adjusting the gating altitude of the laser from $12 \mathrm{~km}$ to another value. This is done by variation of the delays between LGSW and Laser in the master clock.

LGSW High Order Control: The LGS WFS corrects the atmospheric turbulence with the Adaptive Secondary. Tip Tilt modes from the WFS. Therefore the tip tilt slopes of the APD tip-tilt tracker are appended. All the reconstruction is implemented in the Adaptive secondary computing unit.

\subsection{Natural Guide Star WFS control loops}

NGS TT Control: The Tip-tilt tracker unit has a direct connection to the LGS WFS computer and appends the tip tilt slopes to the high order slope vector.

NGS Truth Sensing: The pyramid NGS WFS in a $8 \times 8$ binning mode is used for truth sensing. The 20 first modes are reconstructed and projected into the LGS WFS slope space. The values are subtracted as slope offsets from the LGSW measurement. These calculations are done on the wavefront data handling system.

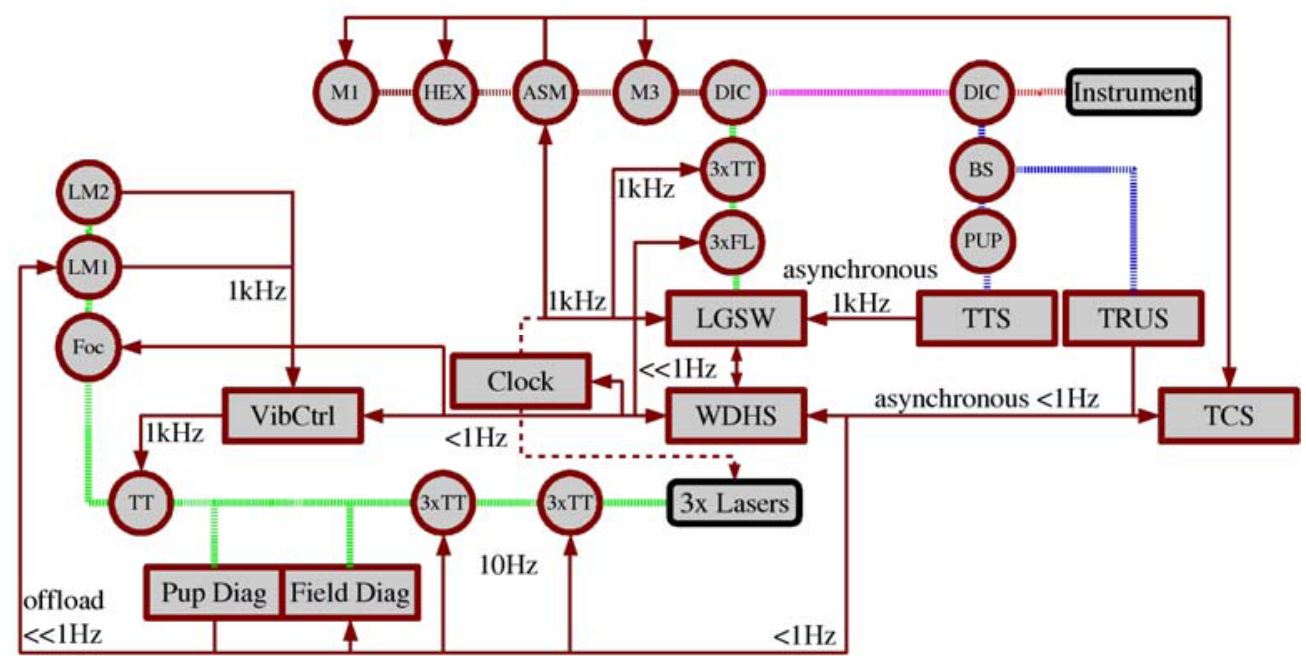

Fig. 9: The scheme shows the control architecture for ARGOS. Circles are optical elements (M1 - primary, HEX - hexapod, ASM - Adaptive Secondary, M3 - Tertirary, Dic - Dichroic, TT - Tip Tilt mirror, BS - Beam splitter, LM - Launch mirror, PUP - Pickup mirror), while rectangles are control units or other hardware. The optical path is drawn in ultra fine dashed lines, the control interaction with solid lines and arrows. The clock is synchronizing the LGSW and Lasers. This hard wired connection is drawn with a dashed line. 


\section{AO CALIBRATION SYSTEM}

A good calibration is an indispensable ingredient for a properly working AO system. The most important calibration of the AO system is interaction between Deformable Mirror and wavefront sensor. The general strategy for the interaction matrix calibration can be summarized as follows: starting from a synthetic matrix, taken over from the NGS AO, this zero order calibration will be refined using artificial light sources in the calibration unit. With this approach a major time on sky is saved both during commissioning and also during operation of the AO. As our adaptive mirror is the LBT's secondary, the light sources for interaction matrix calibration must be located before the deformable mirror in the beam train. As the LBT is a Gregorian design, we are able to place the sources close to the prime focus. In our goal to closely reproduce the laser guide stars with the calibration sources, we use an advanced optical design incorporating a hologram. To bring the optics barrel into prime focus we will install additional swingarms. With those swingarms we ensure that the unit is fully remotely deployable, allowing the operator upon commissioning and routine operation to efficiently calibrate the instrument. This will save us time and long term personnel on the mountain. The swingarm itself is manufactured out of carbon fibre for stiffness, weight and CTE reasons. The realization of the swing-in procedure relies on a precision gearing system and an end-stop defining the location of the optics barrel. By adding some pre-load from the motor the desired precision and repeatability is ensured. A detailed view of the calibration system is given by [22] in this volume.

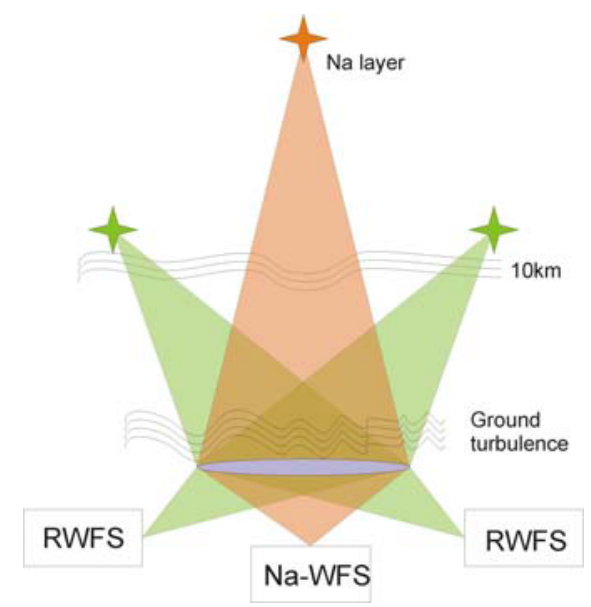

Fig. 10: sketch of the hybrid Rayleigh-Sodium beacon combination. With cleaning up the strong turbulence close to ground with the powerful Rayleigh lasers, an effective larger $r_{0}$ remains from the high altitude turbulence. This part will be detected with the Na-beacon.

\section{A HYBRID LASER SYSTEM FOR DIFFRACTION LIMITED PERFORMANCE}

From the beginning of the project we intended to keep open an upgrade path to diffraction limited performance. Our studies have shown that a hybrid laser system, consisting out of the Rayleigh GLAO facility plus a central sodium guide star would fulfil all our goals. The arrangement could be used either as single conjugated system, or even for future MCAO plans. Thus we foresee space, the injection points, and the associated optics to allow a central $589 \mathrm{~nm}$ laser to be fitted into ARGOS. With current developments in laser technology we see a good chance that we will be able to realize an upgrade within a reasonable time. On the receiver side an optical design has been done for a sodium line wavefront sensor and as well a location in space has been reserved for that. A detailed description and performance calculation is given in [23] and [24] within this proceedings. The major gain that a pre-correction of the turbulence with GLAO delivers comes from an effective increase in $\mathrm{r}_{0}$. Thus for a high layer sodium star correction fewer subapertures are needed and therefore less laser power is required. 


\section{REFERENCES}

Mandel H. et al. "LUCIFER status report: summer 2008”, Proc. SPIE 7014, E.124M, (2008)

Hofmann R. et al. „The cryogenic MOS unit for LUCIFER“, Proc. SPIE 5492, pp. 1243-1253 (2004)

Rigaut F. "Ground conjugated adaptive optics for the ELTs",Beyond conventional adaptive optics, Proc.ESO (2001)

[4] Ellerbroek B., "Multiconjugate adaptive optics for Gemini-South", SPIE 4839, 55, (2003))

[5] Wizinowich et al., "The W. M. Keck Observatory Laser Guide Star System”, PASP (2006)

[6] Bonaccini D. et al."VLT Laser guide star facility ", SPIE 4839 (2003)

[7] Rabien S. et al., "Test performance of the parsec laser system", SPIE 5490 (2004))

[8] Hayano et al. "The laser guide star facility for Subaru telescope", Proc. SPIE 6272 (2006)

[9] D’Orgeville C. et al. , "Gemini north and south laser guide star systems", Proc. SPIE 4494 (2002)

[10] Marchetti E. et al. "MAD on sky results in star oriented mode", Proc. SPIE 7015, pp. 70150F-70150F-12 (2008)

[11] Masciadri E. et al. "Optical turbulence vertical distribution with standard and high resolution at Mt Graham", MNRAS, 404, Issue 1, pp. 144-158 (2010)

[12] Talbot et al. "GLAS: engineering a common-user Rayleigh laser guide star for adaptive optics on the William Herschel Telescope", Proc. SPIE 6272, (2006)

[13] Tokovinin A. et al., "Design of ground-layer turbulence compensation with a Rayleigh beacon", Proc. SPIE 5490, (2004)

[14] Baranec C. "On sky wide field adaptive optics correction using multiple laser guide stars at the MMT", ApJ 693, pp. 1814-1820, (2009)

[15] Quirós-Pacheco F. et al. "First light AO (FLAO) system for LBT: system characterization and performance optimization“, SPIE 7736-128, (2010)

[16] Esposito S. et al. "First light AO (FLAO) system for LBT: final integration and acceptance test results in Europe", SPIE 7736-12, (2010)

[17] Kanneganti S. et al. "ARGOS: a laser constellation for adaptive optics at the LBT", Proc SPIE 7736-161, (2010)

[18] Orban de Xivry G. et al "Wide-field AO correction: the large wavefront sensor detector of ARGOS", Proc. SPIE 7736-199, (2010)

[19] Busoni L. et al. "Final design of the wavefront sensor unit for ARGOS, the LBT's LGS Facility", Proc SPIE 7736208, (2010)

[20] Peter D. "An optimized controller for ARGOS: using multiple wavefront sensor signals for homogeneous correction over the fi eld", Proc SPIE 7736-40, (2010)

[21] Peter D. "Point-spread function reconstruction for the ground layer adaptive optics system ARGOS“, Proc SPIE 7736-177, (2010)

[22] Schwab C. et al. "Calibration strategy and optics for ARGOS at the LBT", Proc SPIE 7736-110, (2010)

[23] Bonaglia et al. "Diffraction limited operation with ARGOS: an hybrid AO system", Proc SPIE 7736-106, (2010)

[24] Hart M. et al. "Diffraction-limited upgrade to ARGOS: the LBT's ground-layer adaptive optics System", Proc SPIE 7736-114, (2010) 